\title{
Invasive Pneumococcal Disease Caused by Non-Vaccine Type Multidrug-Resistant Streptococcus pneumoniae Transmitted by Close Contact in a Healthy Adult
}

\author{
Jeong Rae Yoo ${ }^{1}$, Suhyun $\mathrm{Oh}^{1}$, Jae-Geun Lee ${ }^{1}$, Young Ree Kim² ${ }^{2}$ Keun Hwa Lee ${ }^{3}$, and Sang Taek Heo \\ ${ }^{1}$ Department of Internal Medicine, Jeju National University School of Medicine, Jeju; \\ ${ }^{2}$ Department of Laboratory Medicine, Jeju National University School of Medicine, Jeju; \\ ${ }^{3}$ Department of Microbiology and Immunology, Jeju National University School of Medicine, Jeju, Korea.
}

\begin{abstract}
The incidence of vaccine-type Streptococcus pneumoniae carriage and disease have declined in vaccinated children as well as in unvaccinated children and adults. However, diseases caused by non-vaccine type (NVT) S. pneumoniae are increasing. In this study, we report an invasive pneumococcal disease (IPD) caused by NVT multidrug-resistant (MDR) S. pneumoniae transmitted from a vaccinated infant to an unvaccinated healthy woman, and the clinical characteristics of this serotype. A 29 -year-old previously healthy woman visited our hospital with fever and headache. She had been breastfeeding her baby for 8 months. She was diagnosed with brain abscess and sinusitis caused by S. pneumoniae. Although the patient had no previous exposure to antibiotics, antibiotic susceptibility test identified the pathogen as MDR. The patient's family members were examined using nasopharyngeal swabs for bacterial culture. The serotype of S. pneumoniae identified from the blood, abscess, and sputum of the patient was 15B/C. After investing the patient's family members, we found that the serotype from nasopharyngeal specimen of her baby was the same. We described an invasive MDR pneumococcal disease in an immunocompetent young adult in the community. IPD likely spread to the patient by close contact with her baby, who harbored S. pneumoniae of NVT. The spread of NVT S. pneumoniae in the post-vaccine era has increased in the community, and resistance pattern for S. pneumoniae of $15 \mathrm{~B} / \mathrm{C}$ changed compared to the pre-pneumococcal conjugate vaccine era. The spread of MDR pathogens causing IPD among family members should be monitored.
\end{abstract}

Key Words: Streptococcus pneumoniae, cranial epidural abscess, invasive pneumococcal disease, vaccination

Streptococcus pneumoniae is a common pathogen that causes community-acquired infections, such as upper respiratory infections, pneumonia, meningitis, and sepsis. ${ }^{1}$ Clinical syndromes are classified as noninvasive illnesses, such as contiguous spread from the nasopharynx or skin, and invasive illnesses

Received: August 8, 2019 Revised: September 2, 2019

Accepted: September 3, 2019

Co-corresponding authors: Sang Taek Heo, MD, PhD, Department of Internal Medicine, Jeju National University School of Medicine, 15 Aran 13-gil, Jeju 63241, Korea.

Tel: 82-64-754-8151, Fax: 82-64-717-1131, E-mail: neosangtaek@naver.com and Keun Hwa Lee, PhD, Department of Microbiology and Immunology, Jeju National University School of Medicine, 15 Aran 13-gil, Jeju 63241, Korea.

Tel: 82-64-754-3850, Fax: 82-64-717-1131, E-mail: yomust7@gmail.com

-The authors have no potential conflicts of interest to disclose.

(C) Copyright: Yonsei University College of Medicine 2019

This is an Open Access article distributed under the terms of the Creative Commons Attribution Non-Commercial License (https://creativecommons.org/licenses/ by-nc/4.0) which permits unrestricted non-commercial use, distribution, and reproduction in any medium, provided the original work is properly cited. causing infections in sterile body fluids or bacteremia. ${ }^{2}$ Pneumococci are divided into serotypes based on capsular polysaccharide structures, and 93 serotypes are currently recognized. Serotype distribution varies by age, disease syndrome, and geography. ${ }^{3,4}$ Ever since the licensure and routine administration of polysaccharide-protein pneumococcal conjugate vaccine (PCV), pneumococcal ecology has changed, and the serotype distribution in PCV era may be different. ${ }^{5}$ Non-vaccine type (NVT) strains causing invasive pneumococcal disease (IPD) are circulating worldwide. ${ }^{6}$ The vicious cycle of antibiotic exposure, selection of resistant organisms in the nasopharynx, and transmission of these organisms within the community, which leads to difficult-to-treat infections, has been interrupted to some extent by the introduction and routine use of PCV. Herein, we report an unvaccinated healthy woman with extensive IPD caused by multidrug-resistant (MDR) S. pneumoniae of NVT suspected to have spread from a PCV13 vaccinated infant. 
A 29-year-old healthy woman, who suffered from fever and headache for 3 days, was admitted to our hospital. She was diagnosed with frontal sinusitis and cranial epidural abscess (Fig. 1). S. pneumoniae grew in the blood and tissue specimens from the frontal sinus obtained by a surgical approach. An antibiotic susceptibility test showed an MDR pathogen (Table 1). Based on these results, the patient received levofloxacin (500 mg once daily) and vancomycin (1000 mg twice daily) for 2 weeks on admission and levofloxacin for 4 weeks in an outpatient clinic.

We evaluated the patient's family members (her husband and 9-month-old infant) to detect the origin of the colonizer MDR $S$. pneumoniae. Family members were subjected to nasopharyngeal (NP) swab culture as an outpatient procedure. The patient's blood, sputum, and tissue samples from inflammatory lesions of the frontal, sphenoid, and ethmoid sinus were examined. Specimens were streaked on $5 \%$ sheep blood agar plate, and then incubated for $24-48 \mathrm{hr}$ at $37^{\circ} \mathrm{C}$. In the case of blood, specimens were used after the positive signal in Bact/Alert 3D System (bioMerieux, Marcy-l'Etoile, France). For the identification of isolates, we used standard microbiological techniques using typical colonial appearance, hemolysis, Gram staining, bile solubility, susceptibility to optochin $(1 \mu \mathrm{g})$ discs, and the automated VITEK II system (bioMerieux) with $S$. pneumoniae ATCC 49619 for quality control. According to Clinical and Laboratory Standards Institute (CLSI) guidelines, separate interpretive breakpoints were used to define the resistance of isolates to each antimicrobial agent, ${ }^{7}$ and an isolate resistant to three or more classes of antimicrobial agents was considered MDR. The study protocol was approved by the Institutional Review Board of Jeju National University Hospital (JNUH 1902-007), and informed consent was obtained from all participants (the patient and family members).

S. pneumoniae isolates were re-inoculated onto blood agar plates to identify the molecular serotypes. Isolates with appro- priate properties were serotyped by a sequetyping method, as described previously. ${ }^{8}$ Genomic DNA was extracted from bacterial cells of a colony of pneumococci using heat lysis method. The primers used to amplify and sequence the cps gene were as follows: cps1, 5'-GCA ATG CCA GAC AGT AAC CTC TAT-3', and $c p s 2,5$ '-CCT GCC TGC AAG TCT TGA TT-3'. The cps locus was conserved in all serotypes, and the middle region of the operon contained serotype-specific genes that were used as targets of polymerase chain reaction (PCR)-based serotyping methods. ${ }^{8}$ PCR amplicons were analyzed by $1.5 \%$ agarose gel electrophoresis. A second primer pair targeting the 16S rRNA gene was used to identify the species. Amplicons with the expected $c p s$ band sizes ( 1000 base pairs) were purified using a PCR Purification Kit, according to the manufacturer's instructions. Cycle sequencing was performed using BigDye Sequence Terminator v.3.1 (Applied Biosystems, Foster City, CA, USA), according to the manufacturer's protocol. Amplicon nucleotide sequences were used to search against the GenBank database (https://www.ncbi.nlm.nih.gov/nucleotide/), and the highest BLAST bit score (typically $>98 \%$ identity) was used to identify the serotype. A phylogenetic tree was constructed based on capsular polysaccharide synthesis (cps) gene sequences, using the maximum likelihood method implemented in MEGA 6. ${ }^{9}$ The cps sequence data for $S$. pneumoniae were obtained from NCBI/BLAST (https://blast.ncbi.nlm.nih.gov/ Blast.cgi).

The patient's infant was previously healthy, but had experienced an upper respiratory infection 15 days ago and took medicine from a local clinic. According to the Korean National Immunization Program (NIP), routine PCV 13 vaccination was administrated three times. The husband was also healthy, and did not show any sign and symptom of infection within the past three months. S. pneumoniae was isolated from the NP swab culture of the patient's infant. However, the husband's culture was free of pathogens. When bacterial strain analysis
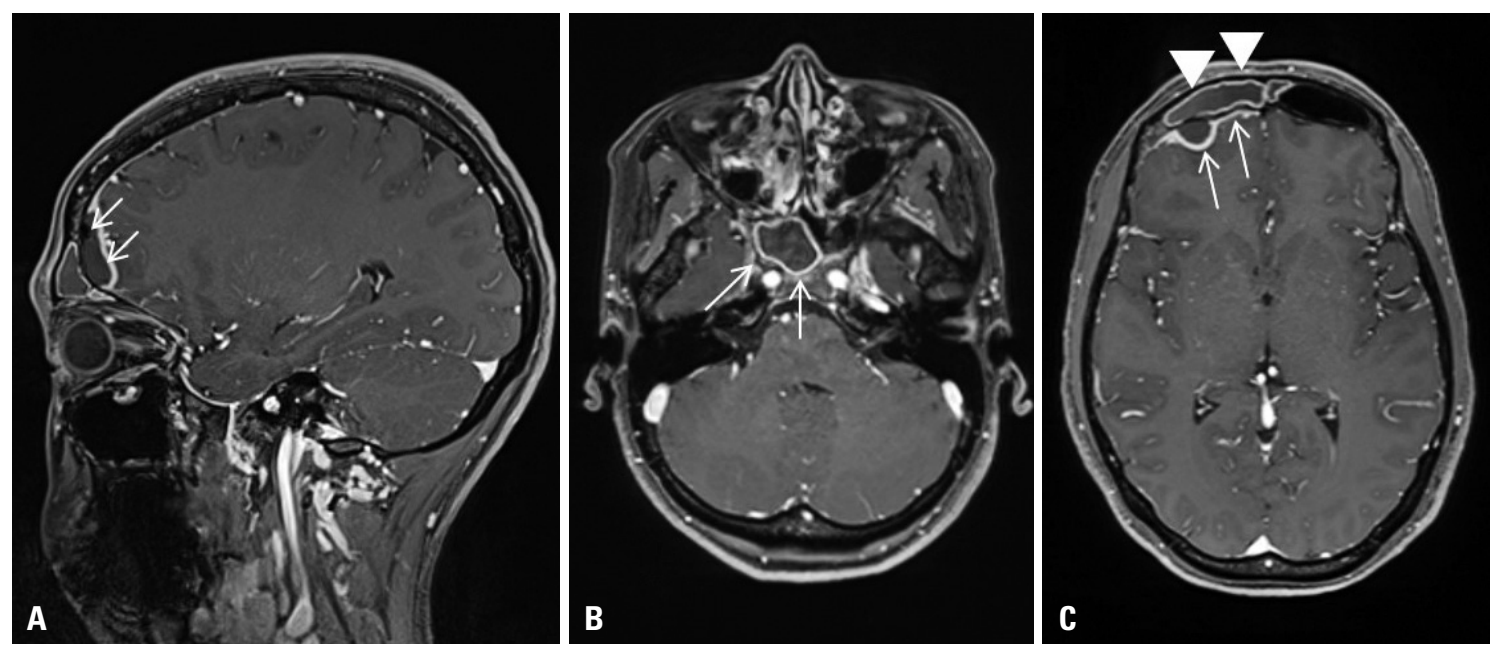

Fig. 1. Enhanced brain magnetic resonance imaging of $1.3 \times 1.0 \times 2.5 \mathrm{~cm}$ sized epidural empyema (A: arrows; $\mathrm{C}$ : arrows), and right frontal (C: arrowheads) ethmoid, and sphenoid sinusitis (B: arrows). Leptomeningeal enhancement with edema on right frontal convexity (C: arrows). 


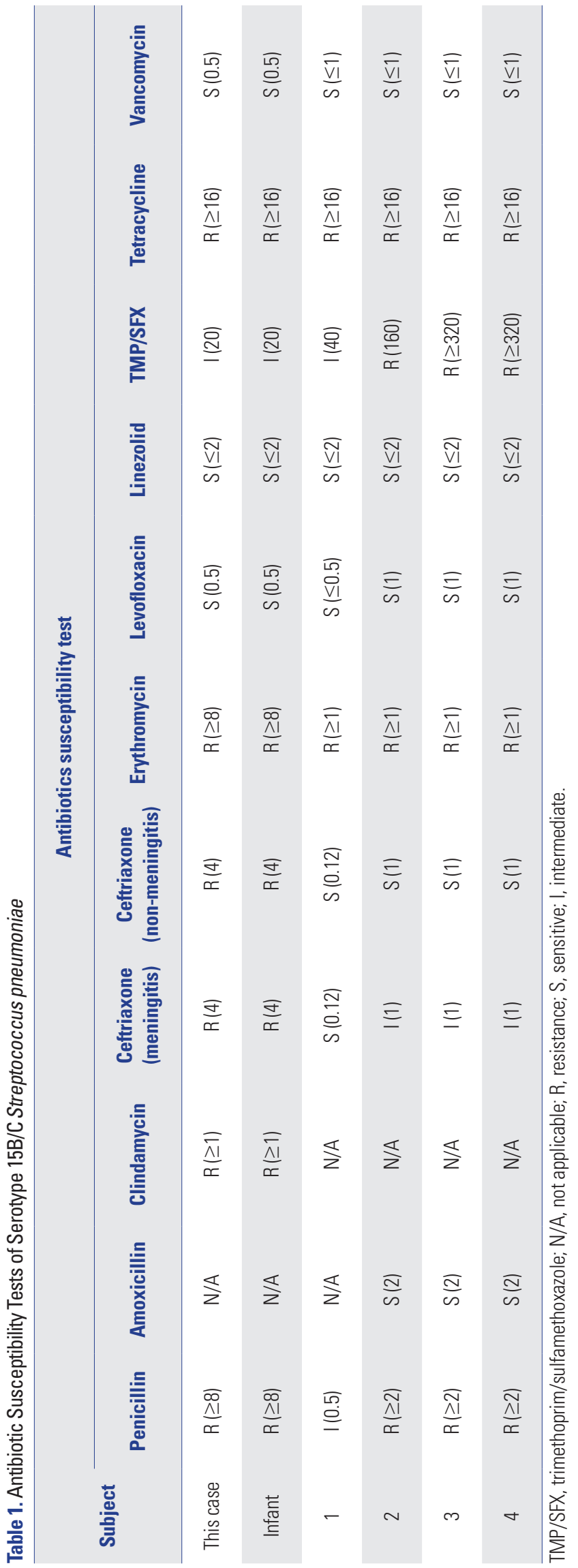

was performed on samples from the patient and her infant, capsule serotype was 15B/C (strain 0556-97/3031-06; GenBank number, KY750640.1/KY750644.1). Antibiotic susceptibility test showed identical results for isolates of the mother and her infant (Table 2), and their sequence types also matched (Fig. 2).

We report on the transmission of an MDR S. pneumoniae of NVT causing cranial epidural abscess, sinusitis, and bacteremia from the nasopharynx of a PCV13-vaccinated infant to a young adult, and changes of antibiotics susceptibilities of serotype 15B/C in PCV era. Pneumococcal colonization is believed to represent the most important source of horizontal spread within the community. ${ }^{10}$ Since children serve as major vectors of pneumococcal transmission, NP colonization may occur in adults via contact with children. ${ }^{11}$ By 1 year of age, $\sim 50 \%$ of children tend to experience at least one episode of pneumococcal colonization. ${ }^{2,12}$ Although the introduction of PCV was followed by a successful reduction in IPD cases for both children and adults, ${ }^{4,11}$ the frequency of PCV serotypes decreased while that of non-PCV serotypes increased among NP carriage pneumococci in the PCV13 era. ${ }^{113}$ A meta-analysis revealed that non-PCV13 serotypes contributed to $42.2 \%$ of childhood IPD cases, and non-PCV13 serotypes, such as 22F, $12 \mathrm{~F}, 33 \mathrm{~F}, 24 \mathrm{~F}, 15 \mathrm{~B}, 15 \mathrm{C}, 23 \mathrm{~B}, 10 \mathrm{~A}$, and 38, were predominant in PCV era. ${ }^{14}$ In addition, an increase in IPD caused by S. pneumoniae of NVT has been reported. ${ }^{13,15}$ In particular, serotype $15 \mathrm{~B} / \mathrm{C}$ is currently among the most prevalent replacement for non-PCV13 serotypes, ${ }^{16}$ and this serotype changes by capsular switching, which involves the emergence of NVT in a prevalent clone by replacement of the capsular polysaccharide locus, such as the switch from serotype $9 \mathrm{~V}$ to $19 \mathrm{~A}$ and from serotype $19 \mathrm{~A}$ to $15 \mathrm{~B} .{ }^{17}$ S. pneumoniae of the patient and her baby in this study were resistant to most antimicrobial agents tested, except linezolid, tigecycline, vancomycin, and levofloxacin, as observed by MDR patterns (Table 1). Additionally, we investigated serotype 15B/C in our previous study. ${ }^{13}$ Four serotypes $15 \mathrm{~B} / \mathrm{C}$ pneumococci were found in two children and two elderly patients (Table 2). All patients had pneumonia. Serotype 15B/C of S. pneumoniae isolates showed non-susceptibility to antibiotic classes (penicillins, macrolides, and tetracyclines) in 2009 and 2010. However, S. pneumoniae isolated from this patient and her infant showed resistance to antibiotic classes (penicillins, lincosamides, macrolides, cephalosporin, and tetracyclines) in 2018. Compared to 2009 and 2010, the resistance pattern for S. pneumoniae of 15B/C changed within the community in 2018. It is important to monitor the frequency of serotype exchanges in order to predict the longterm efficacy of PCV vaccines.

Our study had some limitations. First, we could not definitely conclude that the same serotype had spread from the infant to the mother, since it may have been transmitted from another patient or colonizer with S. pneumoniae of $15 \mathrm{~B} / \mathrm{C}$ serotype. Second, our data may not reflect the nationwide sero- 


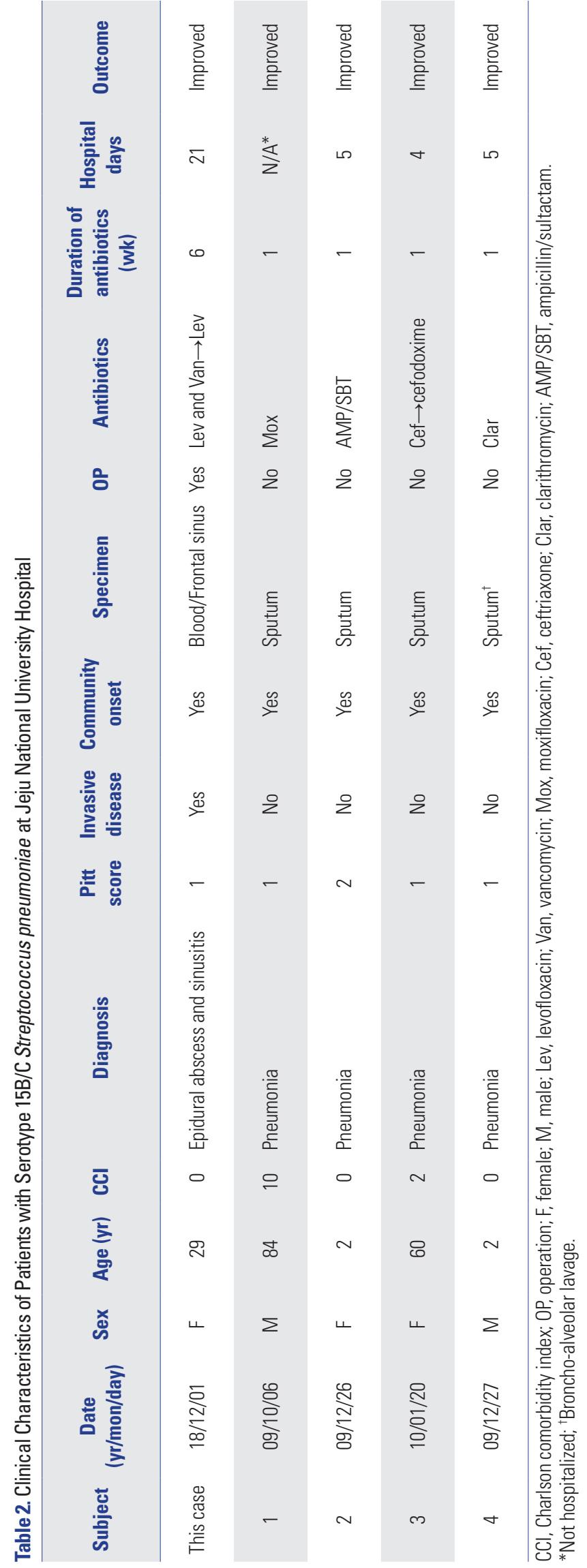

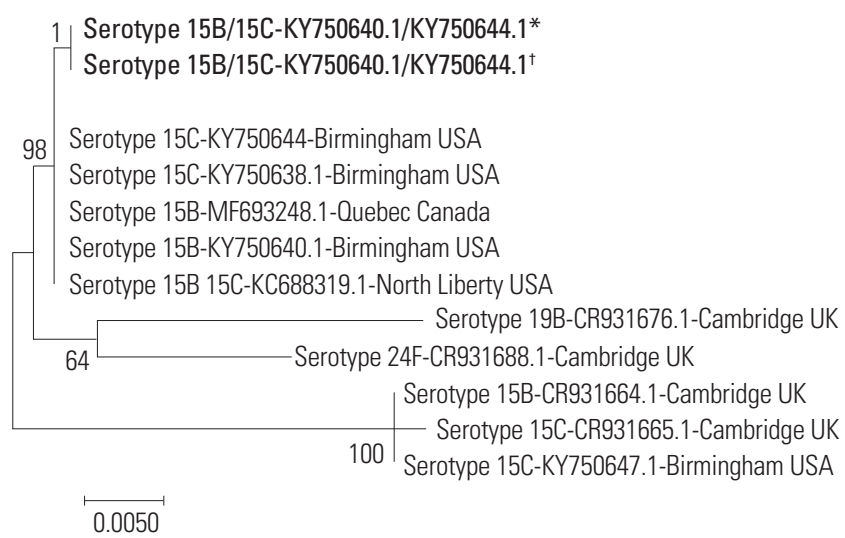

Fig. 2. Phylogenetic tree constructed based on capsular polysaccharide synthesis (cps) gene sequences. The cps sequences generated in this study are shown in bold ( ${ }^{*}$ Patient; ${ }^{\dagger}$ Patient's infant). Scale bar indicates nucleotide substitutions per site.

type and clonal distributions.

IPD of NVT can spread among healthy adults by close contact between family members. Continuous monitoring of antimicrobial resistance and serotype distribution of S. pneumoniae is important for disease management in the Korean population. Further surveillance and studies of serotype distribution in the community are necessary in the PCV 13 era.

\section{ACKNOWLEDGEMENTS}

This work was performed during the author's research year at Yuhan in 2019.

\section{AUTHOR CONTRIBUTIONS}

Conceptualization: Sang Taek Heo. Data curation: Young Ree Kim, Suhyun Oh, Jae-Geun Lee. Formal analysis: Keun Hwa Lee. Investigation: Sang Taek Heo, Jeong Rae Yoo. Methodology: Jeong Rae Yoo, Keun Hwa Lee. Project administration: Sang Taek Heo. Software: Keun Hwa Lee. Supervision: Sang Taek Heo. Validation: Sang Taek Heo. Visualization: Sang Taek Heo. Writing_original draft: Jeong Rae Yoo. Writing_review \& editing: Sang Taek Heo.

\section{ORCID iDs}

Jeong Rae Yoo https://orcid.org/0000-0002-5488-7925 Suhyun Oh Jae-Geun Lee https://orcid.org/0000-0002-5780-2835 https://orcid.org/0000-0003-4915-9584 Young Ree Kim Keun Hwa Lee Sang Taek Heo https://orcid.org/0000-0003-2454-8815 https://orcid.org/0000-0002-2259-2751 https://orcid.org/0000-0001-8947-5069

\section{REFERENCES}

1. Walker CLF, Rudan I, Liu L, Nair H, Theodoratou E, Bhutta ZA, et al. Global burden of childhood pneumonia and diarrhoea. Lancet 2013;381:1405-16.

2. Wessels MR. Streptococcal infections. In: Kasper DL, Fauci AS, 
Hauser SL, Longo DL, Loscalzo J, editors. Harrison's principles of internal medicine. 19th ed. New York: McGraw-Hill Education; 2015. p.963-70.

3. Lee JK, Yun KW, Choi EH, Kim SJ, Lee SY, Lee HJ. Changes in the serotype distribution among antibiotic resistant carriage Streptococcus pneumoniae isolates in children after the introduction of the extended-valency pneumococcal conjugate vaccine. J Korean Med Sci 2017;32:1431-9

4. Bonten MJ, Huijts SM, Bolkenbaas M, Webber C, Patterson S, Gault S, et al. Polysaccharide conjugate vaccine against pneumococcal pneumonia in adults. N Engl J Med 2015;372:1114-25.

5. Choi WS, Choi JH, Kwon KT, Seo K, Kim MA, Lee SO, et al. Revised adult immunization guideline recommended by the Korean society of infectious diseases, 2014. Infect Chemother 2015;47:6879.

6. Slotved HC, Dalby T, Hoffmann S. The effect of pneumococcal conjugate vaccines on the incidence of invasive pneumococcal disease caused by ten non-vaccine serotypes in Denmark. Vaccine 2016;34:769-74.

7. Clinical and Laboratory Standards Institute (CLSI). Performance standards for antimicrobial susceptibility testing. CLSI supplement M100. 27th ed. Wayne (PA): CLSI; 2017.

8. Leung MH, Bryson K, Freystatter K, Pichon B, Edwards G, Charalambous BM, et al. Sequetyping: serotyping Streptococcus pneumoniae by a single PCR sequencing strategy. J Clin Microbiol 2012; 50:2419-27.

9. Tamura K, Stecher G, Peterson D, Filipski A, Kumar S. MEGA6: molecular evolutionary genetics analysis version 6.0. Mol Biol Evol 2013;30:2725-9.

10. Givon-Lavi N, Fraser D, Porat N, Dagan R. Spread of Streptococcus pneumoniae and antibiotic-resistant S. pneumoniae from daycare center attendees to their younger siblings. J Infect Dis 2002;
186:1608-14.

11. Kim YK, LaFon D, Nahm MH. Indirect effects of pneumococcal conjugate vaccines in national immunization programs for children on adult pneumococcal disease. Infect Chemother 2016;48:257-66.

12. Zuccotti G, Mameli C, Daprai L, Garlaschi ML, Dilillo D, Bedogni G, et al. Serotype distribution and antimicrobial susceptibilities of nasopharyngeal isolates of Streptococcus pneumoniae from healthy children in the 13-valent pneumococcal conjugate vaccine era. Vaccine 2014;32:527-34.

13. Yoo JR, Heo ST, Oh H, Oh S, Kim YR, Lee KH. Changes in serotype of Streptococcus pneumoniae after the introduction of the 13-valent pneumococcal vaccine in a homogenous population on Jeju island. Infect Chemother 2019;51:67-72.

14. Balsells E, Guillot L, Nair H, Kyaw MH. Serotype distribution of Streptococcus pneumoniae causing invasive disease in children in the post-PCV era: a systematic review and meta-analysis. PLoS One 2017;12:e0177113.

15. Singleton RJ, Hennessy TW, Bulkow LR, Hammitt LL, Zulz T, Hurlburt DA, et al. Invasive pneumococcal disease caused by nonvaccine serotypes among Alaska native children with high levels of 7 -valent pneumococcal conjugate vaccine coverage. JAMA 2007; 297:1784-92.

16. Dube FS, Ramjith J, Gardner-Lubbe S, Nduru P, Robberts FJL, Wolter N, et al. Longitudinal characterization of nasopharyngeal colonization with Streptococcus pneumoniae in a South African birth cohort post 13-valent pneumococcal conjugate vaccine implementation. Sci Rep 2018;8:12497.

17. Baek JY, Kim SH, Kang CI, Chung DR, Peck KR, Song JH, et al. Emergence of an extensively drug-resistant (XDR) Streptococcus pneumoniae serotype 15A by capsular switching. Int J Med Microbiol 2018;308:986-9. 